\title{
The association between sarcopenic obesity, metabolic risk and the metabolic syndrome in Northern Italian adults
}

\author{
J. Lara ${ }^{1,2}$, M. Powell ${ }^{1}$, M. Siervo ${ }^{1}$, A. Batezzati ${ }^{3}$, A. Leone ${ }^{3}$, A. Tagliabue ${ }^{4}$, A. Spadafranca ${ }^{3}$ \\ and S. Bertolli ${ }^{3}$ \\ ${ }^{1}$ Human Nutrition Research Centre, Institute of Cellular Medicine; Newcastle University. Biomedical Research \\ Building, Campus for Ageing and Vitality. Newcastle Upon Tyne, NE4 5PL, UK., ${ }^{2}$ Department of Applied Sciences, \\ Faculty of Health and Life Sciences, Northumbria University, Ellison Building Room A324, Newcastle upon Tyne, \\ NE1 8ST, UK., ${ }^{3}$ International Center for the Assessment of Nutritional Status (ICANS), Department of Food, \\ Environmental and Nutritional Sciences (DeFENS), University of Milan, Sandro Botticelli, 21, 20133 Milan, Italy. \\ and ${ }^{4}$ Human Nutrition and Eating Disorders Research Centre, Department of Public Health, Experimental and \\ Forensic Medicine, University of Pavia, Via Bassi, 21, 27100 Pavia, Italy
}

The regional distribution of adipose tissue is a well-recognised cardiometabolic risk factor. Previous evidence indicates a positive association between sarcopenic obesity (SO) and the metabolic syndrome (MetSynd) ${ }^{1}$. Here, we evaluated this association defining SO using two indices, the Visceral Adipose Tissue-to-Fat Free Mass ratio (VAT:FFMI) and the Fat Mass index-to-Fat-Free Mass ratio (FFM:FFMI). Body composition was measured using ultrasonography and bioelectrical impedance analysis (BIA) on a sample of 3441 Northern Italian adult men and women. Individuals were defined as SO if they were in the 5th quintile of the distribution for each index. We aimed to examine associations between both indices of SO, metabolic risk and the MetSynd ${ }^{2}$. We also evaluated the value of VAT:FFMI and FMI:FFMI as predictors of metabolic risk and the MetSynd. 689 participants were classified as SO using VAT:FFMI while 681 were classified as SO using the FMI:FFMI ratio. The prevalence of the MetSynd was $65.6 \%$ among SO defined by VAT:FFMI, and 47.6 \% among SO defined by FMI:FFMI. Overall, SO was associated with an increased risk of the MetSynd, particularly with the VAT:FFMI ratio. After adjustment for covariates both indices were associated with important increased risk for MetSynd. Results from logistic regression analysis evaluating the risk of the MetSynd associated with SO are shown in the table.

\begin{tabular}{|c|c|c|c|c|c|c|}
\hline & \multicolumn{3}{|l|}{ VAT:FFMI } & \multicolumn{3}{|l|}{ FMI:FFMI } \\
\hline & $\bar{B}(\mathrm{SE})$ & $\begin{array}{l}\mathrm{OR} \\
(95 \% \mathrm{CI})\end{array}$ & $p$ & $\bar{B}(\mathrm{SE})$ & $\begin{array}{l}\mathrm{OR} \\
(95 \% \mathrm{CI})\end{array}$ & $p$ \\
\hline $\begin{array}{l}\text { Unadjusted Model } \\
\text { SO }\end{array}$ & $2.08(0.094)$ & $\begin{array}{r}\mathbf{8 . 0 3} \\
(6 \cdot 69-9 \cdot 65)\end{array}$ & $<0.001$ & $1.07(0.09)$ & $\begin{array}{r}2.91 \\
(2 \cdot 45-3 \cdot 46)\end{array}$ & $<0.001$ \\
\hline \multicolumn{7}{|c|}{$\begin{array}{l}\text { Adjusted Model } \\
\text { (age, sex, pathology, physical activity, smoking status, marital status, education and employment) }\end{array}$} \\
\hline SO & $1 \cdot 4(0 \cdot 10)$ & $\begin{array}{r}4.06 \\
(3.31-4.97)\end{array}$ & $<0.001$ & $1.45(0 \cdot 11)$ & $\begin{array}{r}\mathbf{4 . 2 5} \\
(3.42-5 \cdot 27)\end{array}$ & $<0.001$ \\
\hline
\end{tabular}

The results of this study show that SO either defined by either VAT:FFMI or FMI:FFMI was an important predictor of cardiometabolic risk among Northern Italian adults. The global increase of the older sector of the population together with the current epidemic of obesity highlights the importance of identifying SO and develop interventions to ameliorate its associated risks.

1. Aubertin-Leheudre M, et al. Obesity 2006; 14, 2277-2283

2. Alberti KG, et al. Circulation 2009; 120, 1640-1645. 